\title{
Confort térmico en interiores y exteriores de espacio educativo en clima cálido semi-seco
}

\author{
Francisco José Martín del Campo Saray \\ Gonzalo Bojórquez Morales
}

Artículo

Afiliación: Francisco José Martín del Campo Saray: Instituto Tecnológico José Mario Molina Pasquel y Henríquez Unidad Académica El Grullo. Jalisco, México.

Gonzalo Bojórquez Morales: Universidad Autónoma de Baja California, Baja California, México.

E-mail: Francisco José Martín del Campo Saray: martindelcampo@elgrullo.tecmm.edu.mx Gonzalo Bojórquez Morales: gonzalobojorquez@uabc.edu.mx

Recibido: 14 de agosto del 2020

Aceptado: 29 de octubre del 2020

Francisco José Martín del Campo Saray Doctor en Arquitectura por la Universidad de Colima. Profesor-investigador del Instituto Tecnológico José Mario Molina Pasquel y Henríquez. Líneas de interés de investigación: Paisaje urbano y espacios públicos, arquitectura bioclimática, habitabilidad ambiental.

\section{Gonzalo Bojórquez Morales}

Doctor en Arquitectura por la Universidad de Colima. Profesor-investigador de la Universidad Autónoma de Baja California y miembro del Sistema Nacional de Investigadores. Líneas de interés de investigación: Confort térmico, habitabilidad ambiental, arquitectura bioclimática.

\section{Resumen:}

En la región de El Grullo, Jalisco, México, existen condiciones de clima cálido semiseco y de acuerdo a revisión de literatura SEMARNAT (2010), este tipo de clima sucede en un $7 \%$ del territorio mexicano. Esto motivó a realizar un estudio de confort térmico en interiores y exteriores de un espacio educativo. El objetivo de la investigación fue estimar el valor de la neutralidad y los rangos de confort de variables del ambiente térmico y del usuario para conocer la sensación y la percepción térmica de sus ocupantes. Se utilizó una metodología descriptiva, no experimental y transversal, previo diagnóstico bioclimático. Para ello, se aplicaron 300 cuestionarios para habitante y ambiente térmico en el periodo cálido y frío. Se utilizó la correlación de Pearson para saber la asociación significativa entre variables meteorológicas y ordinales. Los resultados del estudio indican que en el rubro de sensación térmica en términos de estadística hay una asociación significativa de nivel moderada con la variable de temperatura de bulbo seco, temperatura de globo negro y nivel débil con la humedad relativa. Asimismo, la tolerancia de la temperatura fue significativa en nivel débil con la temperatura de globo negro y radiación solar.

Palabras clave: Aceptación y tolerancia de temperatura, confort térmico, sensación y percepción térmica.

Thermal comfort in interiors and exteriors of educational space in warm semi-dry climate

\begin{abstract}
:
In the region of El Grullo, Jalisco, Mexico, there are conditions of warm semi-dry climate and according to a review of the SEMARNAT literature (2010), this type of climate occurs in $7 \%$ of the Mexican territory. This led to a study of thermal comfort in interiors and exteriors of an educational space. The objective of the research was to estimate the value of neutrality and the comfort ranges of variables of the thermal environment and of the user to know the sensation and thermal perception of its occupants. A descriptive, nonexperimental and cross-sectional methodology was used, after a bioclimatic diagnosis. For this, 300 questionnaires were applied for inhabitants and thermal environment in the hot and cold period. Pearson's correlation was used to know the significant association between meteorological and ordinal variables. The results of the study indicate that in the area of thermal sensation in statistical terms there is a significant association of moderate level with the variable of dry bulb temperature, black globe temperature and weak level with relative humidity. Likewise, the temperature tolerance was significant at a weak level with the black globe temperature and solar radiation.
\end{abstract}

Keywords: Acceptance and tolerance of temperature, thermal comfort, thermal sensation and perception. 


\section{Introducción}

I confort térmico lo define la Norma ISO 7730 (2005) como: "esa condición de la mente en la que se expresa la satisfacción con el ambiente térmico". La primera condición de confort es la neutralidad térmica, lo que significa que la persona no siente demasiado calor ni demasiado frío (Godoy, 2012). Con relación a lo que señala Godoy (2012), se entiende que una persona que se encuentra en neutralidad térmica posee un equilibrio termofisiológico y se adecua a las condiciones meteorológicas que en ese momento suceden, a su vez, presenta una aceptación por el lugar e infiere en su grado de satisfacción personal. Por otra parte, Rein (2013) dice que no existe un nivel definido 0 estándar óptimo de confort, ya que sus resultados dependen de cómo se combinan un conjunto de factores físicos, sociales, culturales y psicológicos del entorno del individuo, así como de su experiencia que condiciona los niveles de satisfacción del ser humano.

Se piensa que las condiciones meteorológicas en un espacio público exterior determinan el uso y permanencia de sus usuarios, a diferencia de los espacios interiores en donde las condiciones de habitabilidad pueden ser controladas, donde se aísla al ser humano de las variables ambientales que le puedan afectar en su desempeño físico o emocional. No obstante, las variables que influyen en el proceso de percepción de la sensación térmica, de acuerdo con Bojórquez (2010) estos son: el metabolismo, la termorregulación humana, el balance térmico, el ambiente térmico y la adaptación térmica humana.

Para efectos de este artículo, el Instituto Tecnológico José Mario Molina Pasquel y Henríquez Campus El Grullo, Jalisco, México, se localiza en el kilómetro 5 Carretera El Grullo-Ejutla, en Jalisco, México. En su creación el Tecnológico Nacional de México sigue guiándose con el Modelo Educativo para el Siglo XXI, el cual orienta el proceso educativo central a la formación de profesionales que impulsen la actividad productiva en cada región del país, la investigación científica, la innovación tecnológica, la transferencia de tecnologías, la creatividad y el emprendedurismo con el fin de alcanzar un mayor desarrollo social, económico, cultural y humano (Carranza et al., 2016). El objeto de estudio fue el Instituto Tecnológico José Mario Molina Pasquel y Henríquez Campus El Grullo que se localiza en la región Sierra de Amula del Estado de Jalisco en México. Este municipio tiene un clima cálido semiseco, la temperatura máxima promedio es de $35^{\circ} \mathrm{C}$, la mínima promedio de $11^{\circ} \mathrm{C}$ y la temperatura media anual es de $23.9^{\circ} \mathrm{C}$ con una precipitación media anual de $900 \mathrm{~mm}$, una altitud de 876 m.s.n.m. y humedad relativa promedio del 25\% (Cuaderno Estadístico Municipal El Grullo, 1996).

Dadas las condiciones climáticas de esta región, se piensa que la sensación y percepción térmica de los usuarios de espacios interiores y exteriores del centro educativo es variable y a su vez influenciada en menor o mayor grado por la etapa estacional del año en que se registran los datos. Las condiciones de clima cálido semiseco del municipio de $\mathrm{EI}$ Grullo, en Jalisco, México y, en específico en estas instalaciones durante en el periodo de verano (junio a septiembre), reducen los niveles de confort térmico, la eficiencia de actividades laborales y académicas de los usuarios que se desarrollan en los interiores del edificio. Con frecuencia se utiliza aire acondicionado, lo cual, representa mayor consumo energético, contaminación ambiental e incremento económico en el pago de suministro de electricidad por parte de la institución con relación a los primeros y últimos meses del año.

De acuerdo con datos de Vargas (2017), la localidad de El Grullo tiene un índice elevado de contaminación atmosférica por la combustión constante de caña de azúcar del ingenio Melchor Ocampo que es uno de los principales productores en el Estado de Jalisco. No existe regulación ni control de la combustión de las parcelas de caña, a esto, se suman los incendios forestales múltiples de la región que se registraron en el año 2017. Se consideró pertinente realizar la investigación en clima cálido semiseco para estudiar los problemas de confort térmico originados por temperaturas en dos periodos estacionales de un año que suceden en los espacios interiores y exteriores del Instituto Tecnológico José Mario Molina Pasquel y Henríquez Campus El Grullo en Jalisco. En el edificio, los materiales de construcción envolventes en algunas áreas no son óptimos para las condiciones climáticas adversas, esto genera estrés térmico y suscita riesgos de salud a sus ocupantes. 
Asimismo, se consideró necesario realizar un estudio que tuviera como fin reducción de gasto energético, optimizar recursos naturales y obtener la estimación de confort térmico que permita establecer la pauta para mejorar las condiciones laborales de los usuarios en el interior y exterior de la institución. Parte de la justificación del estudio es que, hasta el 2018, los estudios sobre confort térmico en México, se desarrollaron en climas cálidos (seco, húmedo y subhúmedo) principalmente en el norte, sur y sureste de México y en climas fríos (semi-frío) en la región de Pachuca, Hidalgo, México. Dicho esto, anteriormente no existía un estudio previo de confort térmico en clima cálido semiseco, tampoco se había desarrollado una investigación donde se estudiara el enfoque adaptativo en espacios educativos interiores y exteriores tanto de forma paralela y simultánea, también se valoró la sensación y percepción térmica de sus ocupantes.

\section{Estado del Arte}

Se revisan investigaciones de autores referentes al tema como (Dear, Nicol, Humpreys, Aluciems, Griffiths, Fanger y Brager), con la finalidad de analizar y sustentar la metodología que se aproxima al estudio. Se recurrió a la revisión crítica de literatura que no tuviera una antigüedad mayor a 10 años junto con los autores clásicos en el tema, los textos se seleccionaron en función de su relevancia. Es decir, que fueran textos mencionados por otros autores, o bien, que abordaran los conceptos que son de interés en la investigación. Los documentos analizados se acomodaron desde el punto de vista temático y por tiempo (de lo más reciente a lo más antiguo) y como segundo orden de lo internacional a lo local, según fuera el caso.

Yang et al. (2017), señalaron que los suecos en las zonas urbanas disfrutan del clima subártico y prefieren los parques para la recreación y las actividades al aire libre con mayor frecuencia en el verano, mencionaron que el microclima al aire libre es importante para determinar la calidad de los espacios exteriores. De acuerdo con lo anterior, Yang et al. (2017), puntualizan la importancia de la percepción térmica de los usuarios sobre los espacios habitables y sobre cómo esto puede afectar la cuestión psicológica y emocional en las respuestas subjetivas a la hora de la aplicación de los cuestionarios. También menciona información sobre el grado de adaptación de los individuos ante los cambios térmicos de un tipo de clima frío donde se asocia esta interacción con el ambiente térmico y su localidad.

Por su parte, Salata et al. (2017), hicieron un estudio transversal de confort térmico en espacios exteriores en el clima templado de Roma, Italia. En la primera parte del cuestionario, los entrevistados respondieron a preguntas personales, mientras que en la segunda parte evaluaron su percepción y preferencia a través de la escala ASHRAE de siete puntos y la escala de Mclntyre, respectivamente. Las líneas de regresión se obtuvieron al elaborar los votos de percepción térmica y al determinar una Temperatura Equivalente Fisiológica (PET) para cada cuestionario. Estas líneas de regresión dieron la posibilidad de calcular los valores de PET neutros: $26.9^{\circ} \mathrm{C}$ para la estación cálida y $24.9^{\circ} \mathrm{C}$ para el frío. Por el contrario, los votos relativos a la preferencia térmica se relacionaron con los correspondientes valores de la temperatura a través de un modelo de curva logística con la función probit: para la estación cálida un valor PET preferido de $24.8^{\circ} \mathrm{C}$, mientras que para la estación fría $22.5^{\circ} \mathrm{C}$.

Desde otra perspectiva, Coccolo et al. (2016) realizaron un estudio en Nagoya, Japón, donde encontraron que la comodidad humana al aire libre fue un parámetro esencial para evaluar la calidad del microclima urbano y proporcionar directrices para el desarrollo urbano sostenible. El objetivo de este trabajo fue obtener una introducción integral al tema de la comodidad humana al aire libre y comprender los procedimientos existentes del entorno urbano. Los procedimientos se subdividieron en tres categorías principales: índices térmicos, índices empíricos e índices basados en ecuaciones lineales. Para cada enfoque, los estudios de casos se presentaron y subdividieron de acuerdo con la clasificación climática de Koeppen (Polar, Frío, Templado, Árido y Tropical).

En el continente americano, Queiroz et al. (2016), realizaron un trabajo donde presentaron los resultados de las encuestas de confort térmico en dos plazas ubicadas 
en la ciudad de Belo Horizonte, Brasil, con clima tropical seco en dos temporadas diferentes. Los parámetros ambientales se compararon con las respuestas registradas durante las encuestas de campo con el fin de evaluar las condiciones de confort térmico. Los entrevistados experimentaron e identificaron posibles procesos de adaptación térmica, de individuos y de comportamiento. Se explica que la percepción y sensación térmica de cada habitante de un espacio interior o exterior se puede considerar influido por cuestiones culturales, sociales, de costumbres y psicológicas. El método de confort adaptativo establece estas premisas entre las variables físicas y de comportamiento psicológico, lo que conlleva a encontrar rangos de confort y temperaturas de neutralidad en los espacios arquitectónicos.

Por su lado, Rupp et al. (2015), en Santa Catarina, Brasil, realizaron una revisión de artículos publicados en los últimos 10 años que examinan las diversas áreas secundarias relacionadas con el confort térmico, discutieron algunos trabajos sobre los modelos de Fanger y de confort térmico adaptativo. Como resultado de la revisión de literatura, fueron clasificados 466 artículos. El artículo en cuestión examinó estándares, experimentos en interiores y ambientes controlados, estudios de campo en edificios de oficinas, educación y residenciales, modelos fisiológicos humanos, estudios de campo al aire libre y semi-exteriores. Ruíz y Correa (2009), realizaron un estudio comparativo en la ciudad de Mendoza, Argentina, con clima cálido húmedo, sobre modelos diversos de confort térmico. Se identificó cuál de ellos puede ser usado apropiadamente para estimar o predecir el confort térmico en espacios públicos con vegetación de ciudades de zonas áridas, cuya configuración se corresponde con el esquema de "ciudad oasis".

Ahora bien, en lo que corresponde a literatura de México, Bojórquez (2010) encontró que las temperaturas de confort térmico en espacios exteriores permiten obtener propuestas de diseño para propiciar el confort térmico de los usuarios. El estudio fue realizado en Mexicali, Baja California Norte, México. Se aplicaron 822 encuestas en las cuales se estimó la temperatura neutra y rangos de confort con el Método de Medias por Intervalo de Sensación Térmica (MIST), propuesto por Gómez Azpeitia (2007). El análisis se realizó para cuatro niveles de actividad: pasiva, moderada, intensa y uno combinado con los tres niveles. Las temperaturas neutras obtenidas mostraron que los sujetos con actividad intensa, con práctica periódica de ejercicio y hábitos apropiados a las condiciones de clima, tuvieron una temperatura de confort térmico similar a aquellos con actividad pasiva.

Por su parte, Ochoa et al (2009), analizaron la utilización de diferentes índices de confort térmico adecuados para la evaluación de las condiciones climáticas en dos estudios de caso representativos de Los Cabos (San José del Cabo, Cabo San Lucas) Baja California Sur con clima desértico semiseco y Cancún, Quintana Roo, México, con clima cálido subhúmedo, en la costa del Caribe Mexicano. Se calcularon las temperaturas de neutralidad (Tn) para temperaturas medias mensuales (To) a lo largo de un año y el Actual Sensation Vote (ASV) horarios para los solsticios y equinoccios de días promedio. Como resultado, se obtuvo que, para Los Cabos, Baja California Sur, México, se manifestaron sensaciones térmicas en verano y otoño que fueron de caluroso a muy caluroso durante el día y noches frías o muy frías en invierno y primavera. En Cancún, Quintana Roo, México, por el contrario, la sensación fue de ligeramente caluroso a caluroso durante prácticamente todo el año, salvo algunas noches frescas en invierno.

Se entiende que, la evaluación de confort térmico ha evolucionado rápidamente y los estudios realizados proponen estrategias de adecuación de las personas al medio ambiente, así como, reducción de flujos de calor, de estrés cotidiano, calidad del aire y espacios apropiados para la productividad, recreación y convivencia del ser humano. Ahora bien, se comprende que la normatividad utilizada en confort térmico es un factor que respalda las estrategias de mejoramiento en espacios arquitectónicos. Además, contribuye con la regulación del ejercicio profesional en beneficio de los habitantes de una ciudad, establece los criterios del diseño de metodología pertinente para la estimación de confort térmico y permite obtener parámetros de confiabilidad de los resultados.

Por su lado, Bojórquez (2010), menciona que el análisis de la normatividad vigente en los estudios de confort térmico permite identificar los alcances y las limitaciones que cada uno de ellos refiere respecto a los usuarios, las condiciones de desarrollo 
de los modelos de confort térmico en los que se basan, las consideraciones de tipo fisiológico y psicológico que presentan, los niveles de arropamiento y actividad y los tipos de espacios arquitectónicos a los que van dirigidas. Este enfoque, tiene una relación estrecha con la investigación, pues el equilibrio obtenido entre las personas y las variables que participan en la evaluación de confort térmico repercute con su desempeño de actividades y el grado de aceptación por un ocupante de un edificio 0 área exterior.

Con relación a lo analizado, se consideró una selección de la regulación aplicada en el ambiente térmico para personas que trabajan con condiciones de clima cálido 0 frío, con ventilación natural o sistemas activos de enfriamiento o calefacción, esto con la finalidad de crear las condiciones propicias para poder desarrollar sus actividades de ocupación humana y que esto no interfiera con situaciones de salud y estrés térmico. Se analizó que las distintas pautas han contribuido con la industria moderna y manufactureras en hacer eficiente el rendimiento laboral de empleados con ambientes térmicos controlados y han propiciado el confort para las personas.

Ver síntesis de la normatividad en tabla 1.

\begin{tabular}{|c|c|c|}
\hline ANSI/ASHRAE 55:2004 & $\begin{array}{l}\text { Condiciones de ambiente } \\
\text { térmico para ocupación } \\
\text { humana. }\end{array}$ & $\begin{array}{l}\text { Especifica las condiciones de } \\
\text { ambiente térmico en las cuales } \\
\text { una fracción determinada } \\
\text { de los ocupantes estaría en } \\
\text { un ambiente térmicamente } \\
\text { aceptable. }\end{array}$ \\
\hline ISO 7726:2002 & $\begin{array}{l}\text { Ergonomía del ambiente } \\
\text { térmico. Instrumentos de } \\
\text { medida de las magnitudes } \\
\text { físicas. }\end{array}$ & $\begin{array}{l}\text { La norma distingue entre } \\
\text { magnitudes físicas básicas y } \\
\text { magnitudes físicas derivadas. }\end{array}$ \\
\hline ISO 8996:2005 & $\begin{array}{l}\text { Ergonomía del ambiente } \\
\text { térmico. Determinación de la } \\
\text { tasa metabólica. }\end{array}$ & $\begin{array}{l}\text { Incluye varios métodos para } \\
\text { estimar la tasa metabólica, } \\
\text { parámetro necesario para } \\
\text { evaluar el confort y el estrés } \\
\text { térmico. }\end{array}$ \\
\hline ISO 9920:2009 & $\begin{array}{l}\text { Ergonomía del ambiente } \\
\text { térmico. Determinación del } \\
\text { aislamiento de la vestimenta. }\end{array}$ & $\begin{array}{l}\text { Establece métodos para } \\
\text { la estimación de las } \\
\text { características térmicas de un } \\
\text { conjunto de ropa (resistencia } \\
\text { a la pérdida de calor seco y de } \\
\text { calor por evaporación). }\end{array}$ \\
\hline ISO 7243:1995 & $\begin{array}{l}\text { Ambientes calurosos. } \\
\text { Estimación del estrés térmico } \\
\text { del hombre en el trabajo basado } \\
\text { en el índice WBGT (Wet Bulbe } \\
\text { Globe Temperature). }\end{array}$ & $\begin{array}{l}\text { El índice WBGT se calcula } \\
\text { a partir de dos ecuaciones, } \\
\text { en función de si el trabajo se } \\
\text { realiza con o sin radiación } \\
\text { solar. }\end{array}$ \\
\hline ISO 9886:2004 & $\begin{array}{l}\text { Ergonomía. Evaluación de la } \\
\text { sobrecarga térmica mediante } \\
\text { mediciones fisiológicas. }\end{array}$ & $\begin{array}{l}\text { Contempla métodos para } \\
\text { medir e interpretar los } \\
\text { parámetros fisiológicos; } \\
\text { temperatura central del } \\
\text { cuerpo, temperaturas } \\
\text { cutáneas, frecuencia cardíaca } \\
\text { y pérdida de masa corporal } \\
\text { debida a la sudoración. } \\
\end{array}$ \\
\hline ISO 7933:2005 & $\begin{array}{l}\text { Ergonomía del ambiente } \\
\text { térmico. Determinación analítica } \\
\text { e interpretación del estrés } \\
\text { térmico mediante el cálculo de } \\
\text { la sobrecarga térmica estimada. }\end{array}$ & $\begin{array}{l}\text { Se describe un método para } \\
\text { la estimación de la tasa de } \\
\text { sudoración y la temperatura } \\
\text { interna que el cuerpo humano } \\
\text { alcanzará en respuesta a las } \\
\text { condiciones de trabajo. } \\
\end{array}$ \\
\hline ISO 11079:2009 & $\begin{array}{l}\text { Ergonomía del ambiente } \\
\text { térmico. Determinación e } \\
\text { interpretación del estrés } \\
\text { debido al frío y se emplea el } \\
\text { aislamiento requerido de la } \\
\text { ropa (IREQ) y los efectos del } \\
\text { enfriamiento local. }\end{array}$ & $\begin{array}{l}\text { Recoge un método basado en } \\
\text { la evaluación del aislamiento } \\
\text { requerido por la ropa para } \\
\text { mantener el equilibrio térmico } \\
\text { del cuerpo. Es aplicable tanto } \\
\text { en locales cerrados como al } \\
\text { aire libre. }\end{array}$ \\
\hline
\end{tabular}




\begin{tabular}{|l|l|l|}
\hline ISO 10551:1995 & $\begin{array}{l}\text { Efecto del ambiente térmico } \\
\text { con el uso de escalas de juicio } \\
\text { subjetivo. }\end{array}$ & $\begin{array}{l}\text { Utiliza un enfoque de } \\
\text { adaptación basado en } \\
\text { respuestas de tipo cerrado a } \\
\text { preguntas en un cuestionario, } \\
\text { que recolectan la información } \\
\text { sobre la sensación térmica } \\
\text { percibida de los individuos } \\
\text { expuestos a distintos } \\
\text { ambientes térmicos. }\end{array}$ \\
\hline ISO 15743:2009 & $\begin{array}{l}\text { Ergonomía del ambiente } \\
\text { térmico. Lugares de trabajo con } \\
\text { frío. Evaluación y gestión de } \\
\text { riesgos. }\end{array}$ & $\begin{array}{l}\text { Presenta unas herramientas } \\
\text { prácticas para la evaluación } \\
\text { del riesgo debido al frío e } \\
\text { incluye: modelos y métodos } \\
\text { para la evaluación y gestión } \\
\text { del riesgo. }\end{array}$ \\
\hline ISO 7730:2006 & $\begin{array}{l}\text { Ergonomía del ambiente } \\
\text { térmico. Determinación analítica } \\
\text { e interpretación del bienestar } \\
\text { térmico mediante el cálculo de } \\
\text { los índices PMV Y PPD y los } \\
\text { criterios de bienestar térmico } \\
\text { local. }\end{array}$ & $\begin{array}{l}\text { los ambientes témicos } \\
\text { moderados. La sensación } \\
\text { térmica experimentada por un } \\
\text { ser humano está relacionada, } \\
\text { principalmente, con el } \\
\text { equilibrio térmico global de su } \\
\text { cuerpo. }\end{array}$ \\
\hline
\end{tabular}

En relación con las normas presentadas, se estableció un criterio de selección para el diseño del cuestionario y registro de datos en campo. Cabe mencionar que el uso de la normatividad en la investigación favoreció la comprensión y asimilación de los contenidos. Antes de pasar a la etapa de metodología se señala que el objetivo general de la investigación fue estimar, con el enfoque adaptativo, el valor de neutralidad y los rangos de confort de cada una de las variables físicas del ambiente térmico (temperatura de bulbo seco TBS, temperatura de globo negro TGN, radiación solar RS, humedad relativa HR y velocidad de viento $\mathrm{VV}$ ) y las variables complementarias (actividad metabólica AM y aislamiento térmico del vestido ATV). Esto con el fin de determinar la sensación y percepción térmica de usuarios en espacios interiores y exteriores del Instituto Tecnológico José Mario Molina Pasquel y Henríquez Campus El Grullo, Jalisco, México, en bioclima cálido semiseco en dos periodos estacionales del año.

\section{Metodología}

En este apartado se señalaron los aspectos metodológicos para la investigación, se describieron sus etapas, el proceso de captura y la forma de análisis de los datos obtenidos. Las fases fueron el diseño de la investigación, caso de estudio, áreas de estudio, diseño de muestreo y de las cédulas de información, descripción de componentes de las cédulas de información, horarios de aplicación, periodicidad y duración de llenado de cédulas de información, aplicación de la cédula de información, selección, proceso de captura y análisis de datos, instrumentos de medición de variables meteorológicas y registro de base de datos. El diseño de investigación se definió por las características de sus variables y se aplicó una metodología de tipo descriptiva, no experimental y transversal con el fin de conocer la sensación y percepción térmica de usuarios en espacios interiores y exteriores del centro educativo.

Con el fin de llevar a cabo esta investigación, se propuso que el objeto de estudio fueran los usuarios que ocupan los espacios interiores y exteriores del Instituto Tecnológico José Mario Molina Pasquel y Henríquez Campus El Grullo, Jalisco, México, que se ubica en el kilómetro 5 de la carretera El Grullo-Ejutla en la región Sierra de Amula del Estado de Jalisco. Este municipio tiene un clima cálido semiseco, la temperatura máxima promedio es de $35^{\circ} \mathrm{C}$, la mínima promedio de $11^{\circ} \mathrm{C}$ y la temperatura media anual es de $22.3^{\circ} \mathrm{C}$ con una precipitación media anual de $900 \mathrm{~mm}$, una altitud de 935 m.s.n.m., humedad relativa promedio del $35 \%$ y coordenadas de $19^{\circ} 51^{\prime} 18.14^{\prime \prime} \mathrm{N}$ y $104^{\circ}$ 13' 56.67" O. Ver ubicación e imágenes del lugar. Ver figura 1 y 2. 


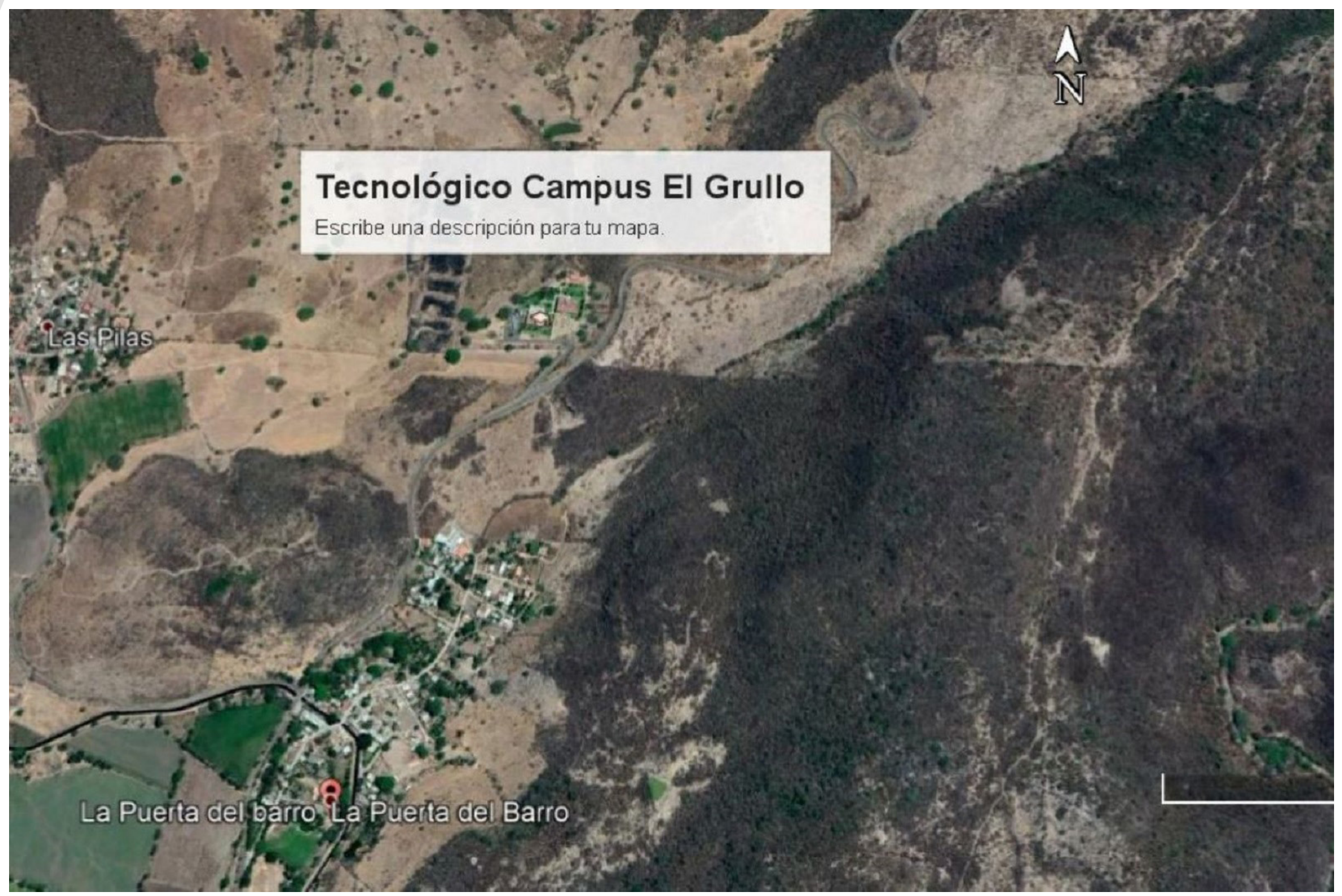

Figura 1. Ubicación de Tecnológico Campus El A

Figura 2. Imagen aérea de Tecnológico Campus $V$

Fuente: Imagen obtenida de Google.

Fuente: Imagen obtenida de Google.

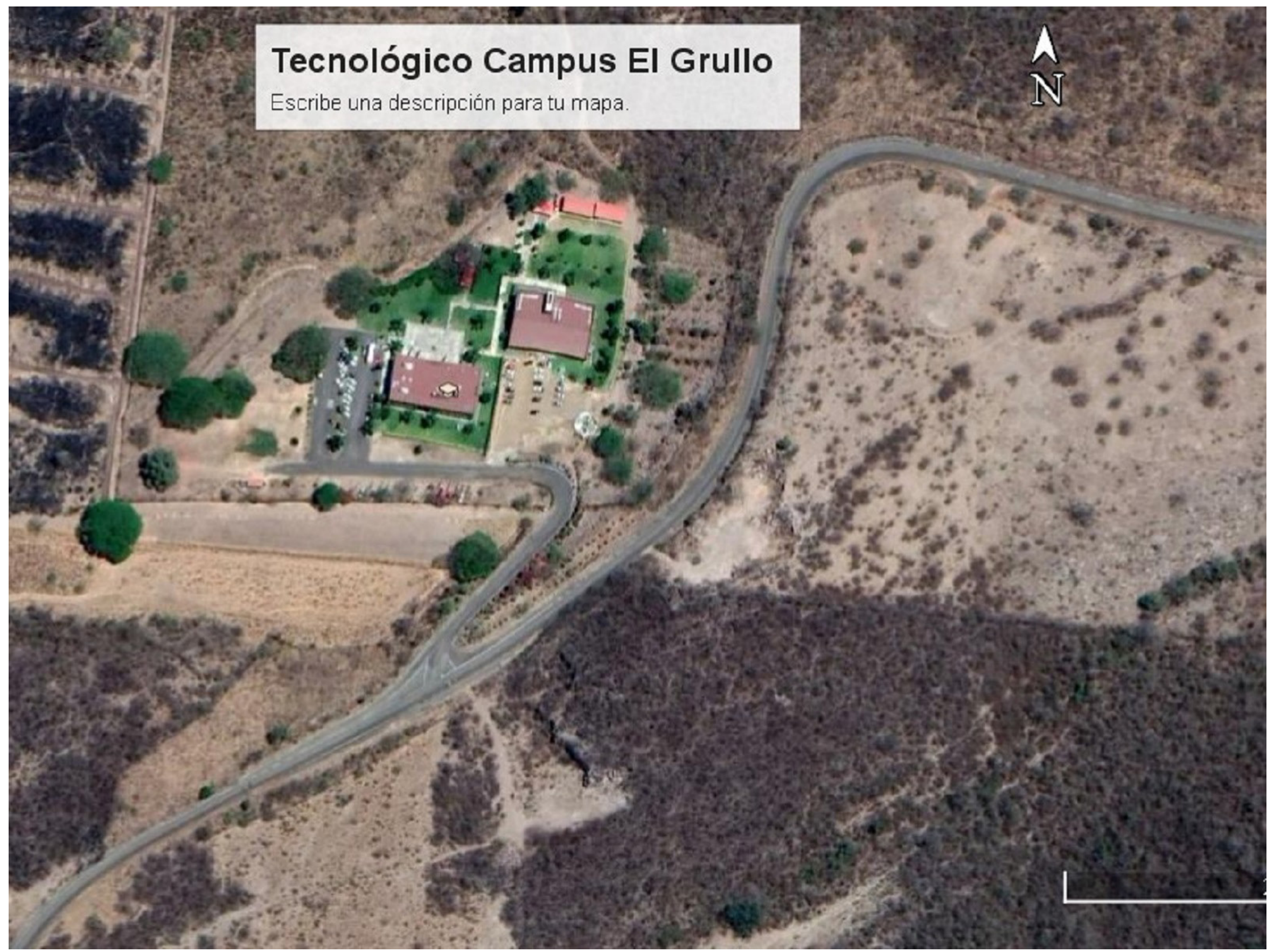


Sobre el diseño del muestreo y de acuerdo al diagnóstico bioclimático de las horas de confort para la población de El Grullo, Jalisco, México, según datos del Sistema Meteorológico Nacional y el análisis de las áreas de estudio, el muestreo se realizó de forma aleatoria con usuarios de espacios interiores y exteriores del Instituto Tecnológico José Mario Molina Pasquel y Henríquez Campus El Grullo, Jalisco, México, con edades entre 18 y 60 años de edad, sin características de enfermedades cardiovasculares, enfermedades neurológicas, mujeres embarazadas o en periodo de lactancia, estas especificaciones se determinaron con base en criterios de la norma ISO 7243:1989 (Estrés térmico en personas con trabajo en ambientes cálidos) para respuestas de sensación y percepción térmica. Se realizó un total de 300 cédulas de información de usuario y ambiente térmico, 149 para el periodo cálido y 151 para el periodo frío. Con relación a los datos de variables físicas de ambiente térmico, se registraron en una bitácora de control interna de información específica para datos meteorológicos y complementarios. Ver figura 3 y 4.

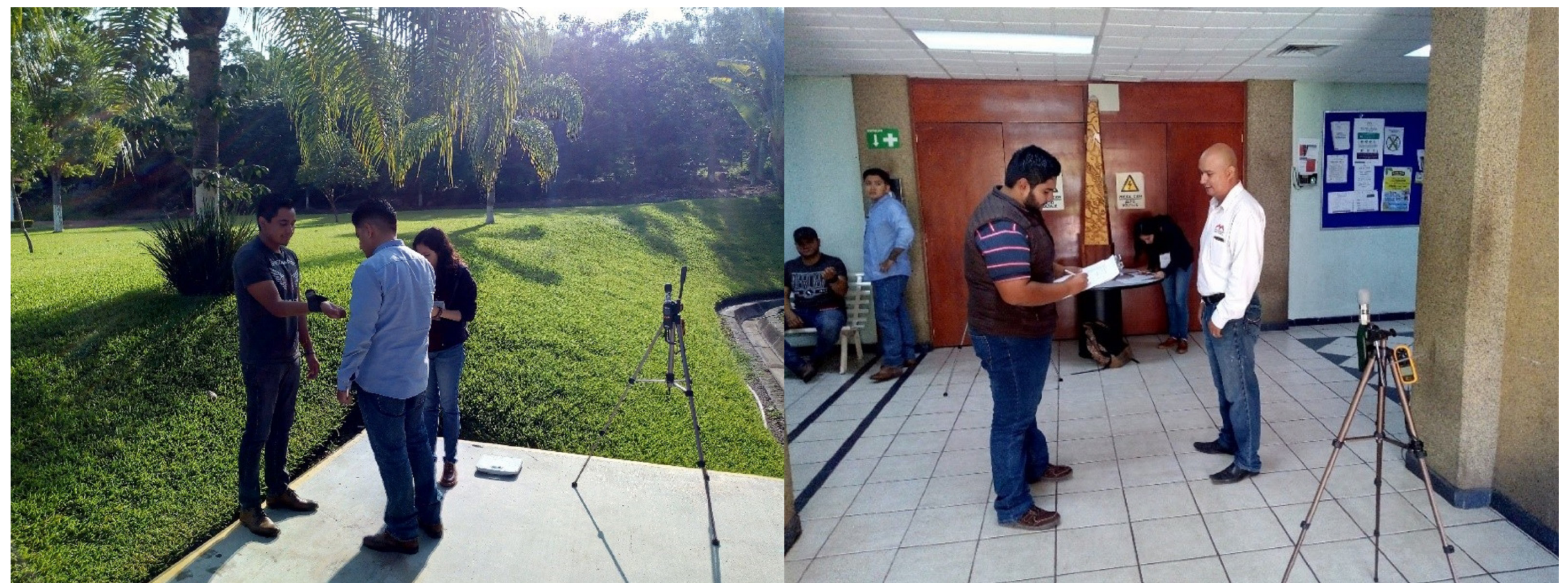

Figura 3. Muestreo en espacio exterior de Tecnológico Campus El Grullo. Fuente: Elaboración propia.

Figura 4. Muestreo en espacio interior de Tecnológico Campus El Grullo. Fuente: Elaboración propia.
A Posteriormente, se describió la cédula de información de usuario y de ambiente térmico, la conformación de sus partes y cuáles fueron las condiciones que se tienen para su aplicación. Se señaló que las cédulas de información fueron realizadas y adecuadas a las necesidades de la investigación, con base en estudios de confort térmico realizados por Bojórquez (2010) y Rincón et al. (2015). Asimismo, se hace referencia a que cada apartado fue subdividido por filas y con un número específico, con la intención de tener un control óptimo para el vaciado de datos y que el análisis e interpretación de estos no fueran confusos y facilitaran la etapa de estadística.

La cédula de información usuario y ambiente térmico contuvo lo siguiente: 01) datos de control, 02) datos del entrevistado, 03) datos del espacio y condiciones de aplicación, 04) sensación térmica y percepción térmica y 05) ambiente térmico. Los apartados tuvieron indicadores o variables según fuera el caso, se tuvieron para registro y control de reactivos de la cédula de información, mostraron los conceptos y si correspondía a un indicador (I) o una variable $(\mathrm{V})$, además, se señaló si la fuente de información se llenaría en campo $(C)$ o en gabinete $(G)$. Con estos componentes, una vez que se capturaron los datos, se analizó la asociación de variables para obtener resultados y saber si estadísticamente fueron significativos.

La aplicación de la cédula de información, medición de variables y recopilación de datos se realizó en un máximo de 10 minutos. Con base en las condiciones climáticas por periodo anual que dan lugar a la zona de estudio y que se manifestaron a lo largo de un año típico, se sugirió realizar estudio de confort térmico de usuarios en espacios interiores y exteriores del instituto durante dos periodos estacionales específicos. Estos fueron agosto y noviembre del 2019, que comprendió el periodo cálido y frío respectivamente. Los horarios en que se aplicaron los cuestionarios fueron en horarios alternos matutino y vespertino de 3 horas continuas, donde se inició a las 07:00 horas y se finalizó a las 22:00 horas. Acerca de los instrumentos de medición para variables meteorológicas se utilizaron dos medidores de estrés térmico (mca. Extech, TGBH mod. HT30), dos anemómetros (mca. Extech, mod. AN25), dos trípodes (mca. Amazon's Choice, mod. 
Figura 5. Vista de instrumentos de medición de variables meteorológicas.

Fuente: Elaboración propia.
WT3111H), dos piranómetros (mca. Tenmars, mod.TM206), un psicrómetro digital (mca. Extech, mod. RH401) y dos registradores de datos (DataLogger mca. Extech, mod. RHT50). Ver figura 5.
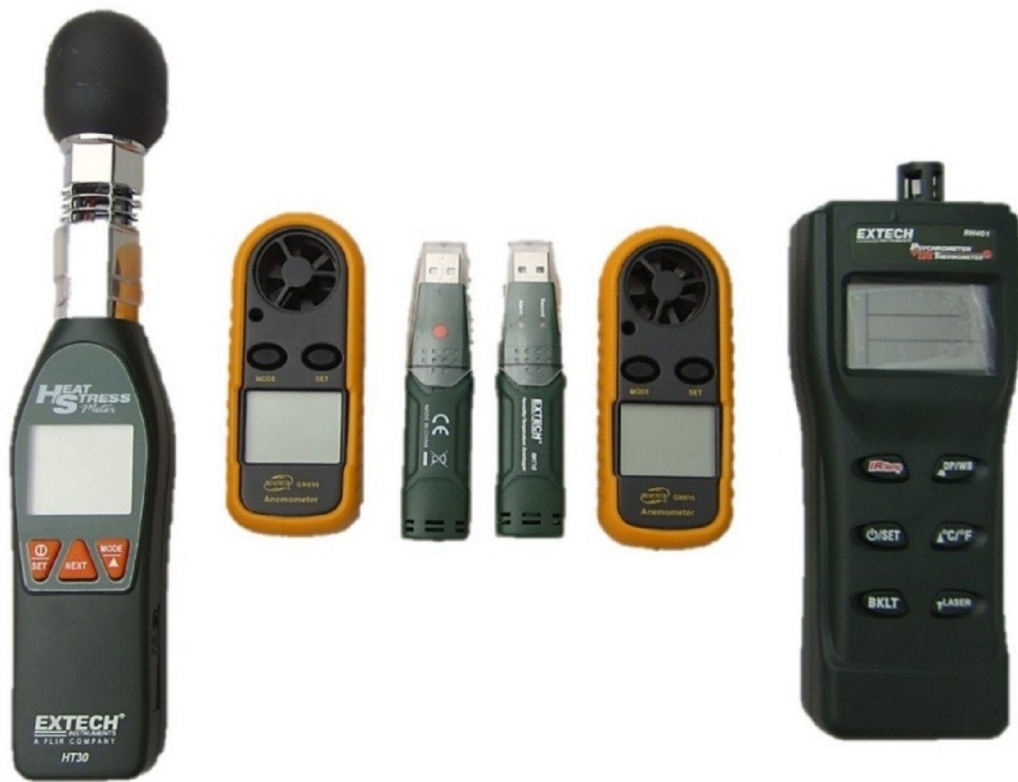

El periodo de muestreo se aplicó del día 09 al 13 de septiembre para el periodo cálido y del 25 al 29 de noviembre del 2019 para el periodo frío, en horarios alternados de tres horas continuas de las 08:00 a las 22:00 horas. Se registraron variables meteorológicas, del usuario y del espacio interior y/o exterior de las instalaciones del Tecnológico José Mario Molina Pasquel y Henríquez Campus El Grullo, Jalisco, México. El montaje de los instrumentos de medición (medidor de estrés térmico, anemómetro digital y piranómetro) se colocaron sobre tripiés a una altura de $1.30 \mathrm{~m}$ con respecto al nivel de suelo según recomendaciones de la Organización Meteorológica Mundial (2014). El psicrómetro digital se colocó a la altura requerida para registrar datos de radiación infrarroja de diversos materiales constructivos y naturales de pisos, techos, muros y mobiliario que se encontraran circundantes a los espacios interiores y exteriores de las áreas de muestreo. Ver figura 6 y 7.

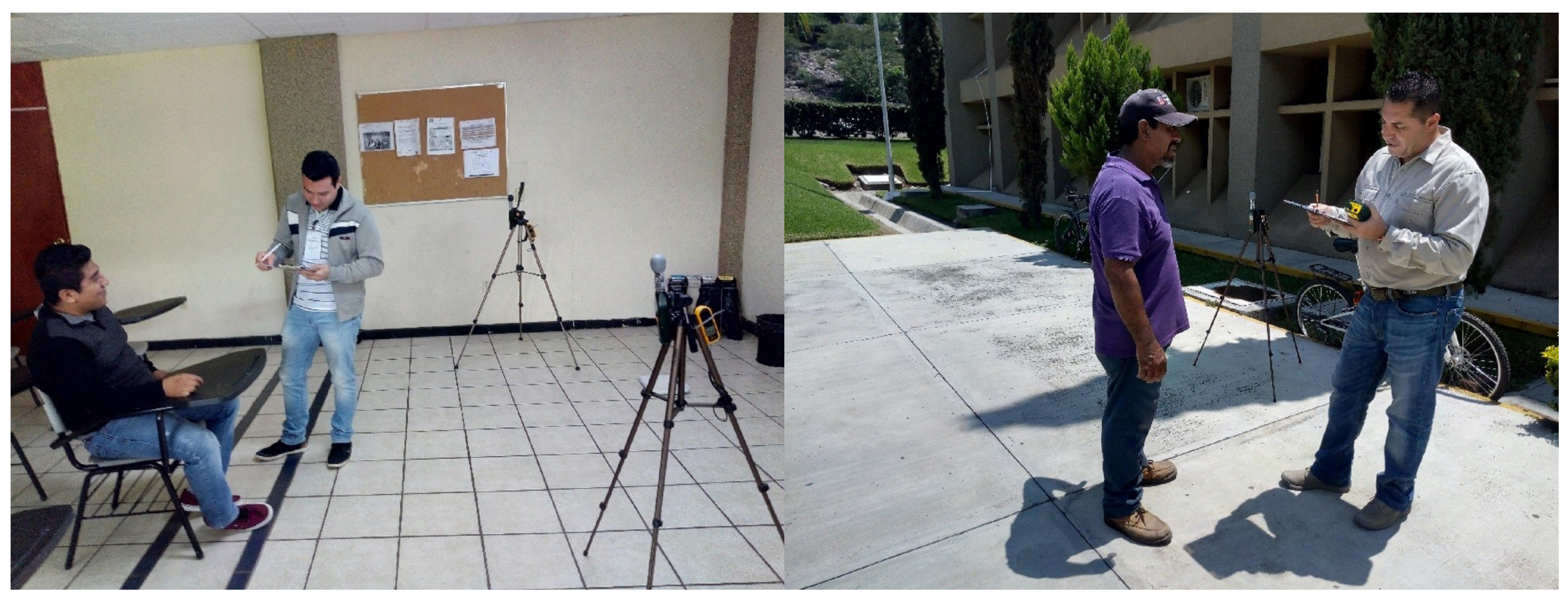

Figura 6. Montaje de instrumentos de medición de variables meteorológicas.

Fuente: Elaboración propia.

Figura 7. Muestreo y medición de variables meteorológicas.

Fuente: Elaboración propia.
Con respecto al registrador de datos, este se ubicó en un área representativa del espacio interior y exterior respectivamente, en horario de 07:00 a 22:00 horas en los días de muestreo. Como complemento del registro de datos para usuario se utilizó un termómetro digital, una báscula, flexómetro, aparato para medición de presión sanguínea y pulso cardíaco. Ver figura 8 y 9 .

Para los muestreos señalados se contó con el apoyo de cuatro personas que se ubicaron de manera estratégica en los espacios diversos del centro educativo para realizar el muestreo. De forma simultánea se registraron datos de las variables del espacio, del usuario y meteorológicas. Una vez obtenidos los resultados del periodo cálido y periodo frío, se codificaron registros para facilitar la captura de las cédulas de información. Se capacitó al personal de apoyo para la captura adecuada de las variables y se utilizó una base de datos exclusiva para la investigación con el programa de Microsoft Excel de 
Figura 8. Montaje de registrador de datos en exterior.

Fuente: Elaboración propia

Figura 9. Montaje de registrador de datos en interior.

Fuente: Elaboración propia.

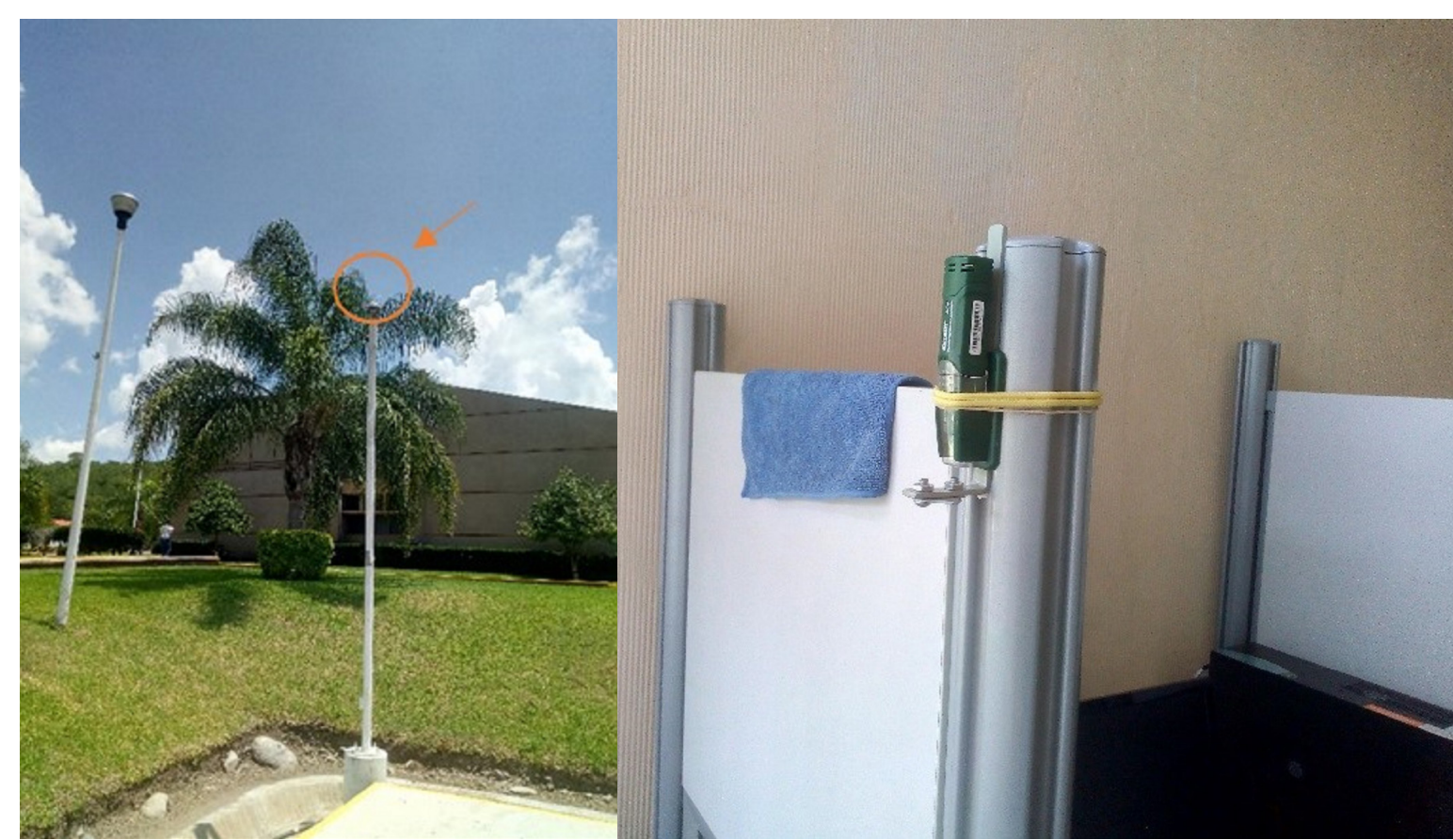

Windows. A su vez, se trasladó la información recabada al programa estadístico SPSS para el análisis y asociación de variables ordinales y nominales.

Respecto al análisis de datos, se aplicó la regresión lineal múltiple con las variables meteorológicas y ordinales, obtenidos estos datos se utilizó el coeficiente de correlación de Pearson, ya que los coeficientes en este tipo de correlación fueron la expresión numérica que indicó el grado de relación lineal existente entre variables cuantitativas o de intervalo y ordinales. A su vez, son los datos que aportaron directamente al estudio de confort térmico. Ya que, el coeficiente de correlación de Pearson y Spearman es un índice cuyos valores absolutos oscilan entre $-1 \mathrm{y}+1$, su magnitud indica el grado de asociación entre las variables, el valor $r=0$ indica que no existe relación entre ellas. Los valores $+/-1$ son indicadores de una correlación perfecta, es positiva al crecer 0 decrecer $\mathrm{x}$, crece $\mathrm{o}$ decrece $\mathrm{y}, \mathrm{o}$ negativa al crecer 0 decrecer $\mathrm{x}$, decrece $\mathrm{o}$ crece $\mathrm{y}$. La matriz de correlación se calculó mediante el uso del programa estadístico SPSS para Windows.

\section{Resultados}

El propósito de la investigación fue presentar resultados de confort térmico en clima cálido semiseco en interiores y exteriores del Instituto Tecnológico José Mario Molina Pasquel y Henríquez Campus El Grullo, con la finalidad que este tipo de estudios resulte de interés y beneficio para la región de Sierra de Amula y Sierra Costa Sur de Jalisco, México. La aplicación del estudio se llevó a cabo en la localidad de El Grullo, Jalisco, México, en dos periodos, el cálido y el frío. Se analizaron las variables de ambiente térmico, de habitante y de espacio. En relación con las variables de ambiente térmico y habitante se recolectaron 300 cédulas de información para ambos periodos, 149 para el periodo cálido y 151 que correspondieron al periodo frío, asimismo, se registraron cédulas de información independientes para el rubro de las variables meteorológicas.

También, se elaboraron tablas y gráficos correspondientes a los resultados del estudio de confort térmico en espacios interiores y exteriores del campus El Grullo por periodos. Asimismo, se identificó la asociación de variables ordinales con variables meteorológicas y de usuario, con el método de regresión múltiple y la correlación de Pearson para saber el nivel de significatividad entre cada una de las variables involucradas. Los resultados que se obtuvieron se organizaron de lo genérico a lo específico en el orden siguiente:

- Genérico en espacios interiores y exteriores periodo cálido: rangos de edad, sexo, lugar de origen, permanencia, área de aplicación y horarios de aplicación de muestreo.

Específico en espacios interiores periodo cálido: actividad metabólica (AM), tipo de vestimenta (TV), sensación térmica (ST), sensación de humedad (SH), sensación de viento $(\mathrm{SV})$, preferencia térmica $(\mathrm{PT})$, preferencia de humedad $(\mathrm{PH})$, preferencia de viento (PV), aceptación de la temperatura (AT) y tolerancia de la temperatura 
Tabla 1. Asociación de sensación térmica con variables meteorológicas en interiores periodo cálido.

** La correlación es significativa al nivel 0,01 (bilateral).

* La correlación es significante al nivel 0,05

(bilateral).

Fuente: Elaboración propia.
Específico en espacios exteriores periodo cálido: actividad metabólica (AM), tipo de vestimenta (TV), sensación térmica (ST), sensación de humedad (SH), sensación de viento (SV), sensación de radiación (SR), preferencia térmica (PT), preferencia de humedad $(\mathrm{PH})$, preferencia de viento $(\mathrm{PV})$, preferencia de radiación $(\mathrm{PR})$, aceptación de la temperatura (AT) y tolerancia de la temperatura (TT).

- Correlación de Pearson y dispersión lineal entre variables ordinales y variables meteorológicas en espacios interiores y exteriores periodo cálido: sensación térmica, preferencia térmica, aceptación de la temperatura y tolerancia de la temperatura con temperatura de bulbo seco (TBS), temperatura de globo negro (TGN), radiación solar (RS), humedad relativa (HR) y velocidad de viento (VV).

- Correlación de Pearson y dispersión lineal entre variables ordinales y variables de usuario en espacios interiores y exteriores periodo cálido: sensación térmica, preferencia térmica, aceptación de la temperatura y tolerancia de la temperatura con actividad metabólica y tipo de vestimenta.

Para los resultados representativos del manuscrito, solo se presentan algunas tablas aleatorias de correlación de Pearson entre variables ordinales y variables meteorológicas y de usuario, de espacios interiores y exteriores en periodo cálido y periodo frío. Ver tablas 1 a la 4 . Para el periodo cálido en los espacios interiores el $46.8 \%$ de los entrevistados manifestaron la variable de sensación térmica en la escala de "ni calor ni frío", el 38.3\% la escala de "poca" sensación de humedad y el $80.9 \%$ la escala de "nada" en sensación de viento. Sin embargo, en relación con la preferencia térmica la escala que resultó mayor fue "más frío" con el $36.2 \%$, la escala de "nada" con un $41.5 \%$ para la preferencia de humedad y la escala de "media" con un $33.0 \%$ para la preferencia de viento. En lo que refiere a la aceptación de la temperatura la escala de "aceptable" fue la mayor con el $54.3 \%$ de los registros y la tolerancia de la temperatura fue la escala de "tolerable" en el $64.9 \%$ de los casos.

\begin{tabular}{|c|c|c|c|c|c|c|c|}
\hline & & $\begin{array}{l}\text { U04.1 } \\
\text { Sensación } \\
\text { térmica }\end{array}$ & \begin{tabular}{|l} 
U05.01 \\
Temperatura \\
de bulbo seco
\end{tabular} & $\begin{array}{l}\text { U05.02 } \\
\text { Temperatura } \\
\text { de globo } \\
\text { negro }\end{array}$ & $\begin{array}{l}\text { U05.04 } \\
\text { Radiación } \\
\text { solar }\end{array}$ & $\begin{array}{l}\text { U05.05 } \\
\text { Humedad } \\
\text { relativa }\end{array}$ & $\begin{array}{l}\text { U05.07 } \\
\text { Velocidad de } \\
\text { viento }\end{array}$ \\
\hline \multirow{3}{*}{ U04.1 Sensación térmica } & Correlación de Pearson & 1 &, $571^{* *}$ & , 410** &, $306^{* *}$ & $-0,12$ & $-0,087$ \\
\hline & Sig. (bilateral) & & 0 & 0 & 0,003 & 0,247 & 0,404 \\
\hline & $\mathrm{N}$ & 94 & 94 & 94 & 94 & 94 & 94 \\
\hline \multirow{3}{*}{$\begin{array}{l}\text { U05.01 Temperatura de bulbo } \\
\text { seco }\end{array}$} & Correlación de Pearson &, $571^{* *}$ & 1 &, $574^{* *}$ &, $357^{*+*}$ &,$- 554^{* *}$ & $-0,107$ \\
\hline & Sig. (bilateral) & 0 & & 0 & 0 & 0 & 0,303 \\
\hline & $\mathrm{N}$ & 94 & 94 & 94 & 94 & 94 & 94 \\
\hline \multirow{3}{*}{$\begin{array}{l}\text { U05.02 Temperatura de globo } \\
\text { negro }\end{array}$} & Correlación de Pearson &, $410^{* *}$ &, $574^{* * *}$ & 1 & 0,121 &,$- 237^{+}$ & 0,065 \\
\hline & Sig. (bilateral) & 0 & 0 & & 0,244 & 0,021 & 0,534 \\
\hline & $\mathrm{N}$ & 94 & 94 & 94 & 94 & 94 & 94 \\
\hline \multirow{3}{*}{ U05.04 Radiación solar } & Correlación de Pearson &, $306^{* *}$ &, $357^{* *}$ & 0,121 & 1 &,$- 230^{*}$ & $-0,017$ \\
\hline & Sig. (bilateral) & 0,003 & 0 & 0,244 & & 0,026 & 0,871 \\
\hline & $\mathrm{N}$ & 94 & 94 & 94 & 94 & 94 & 94 \\
\hline \multirow{3}{*}{ U05.05 Humedad relativa } & Correlación de Pearson & $-0,12$ &,$- 554^{* *}$ &,$- 237^{\star}$ &,$- 230^{*}$ & 1 & 0,046 \\
\hline & Sig. (bilateral) & 0,247 & 0 & 0,021 & 0,026 & & 0,659 \\
\hline & $\mathrm{N}$ & 94 & 94 & 94 & 94 & 94 & 94 \\
\hline \multirow{3}{*}{ U05.07 Velocidad de viento } & Correlación de Pearson & $-0,087$ & $-0,107$ & 0,065 & $-0,017$ & 0,046 & 1 \\
\hline & Sig. (bilateral) & 0,404 & 0,303 & 0,534 & 0,871 & 0,659 & \\
\hline & $\mathrm{N}$ & 94 & 94 & 94 & 94 & 94 & 94 \\
\hline
\end{tabular}


Para el periodo cálido en los espacios exteriores el $33.3 \%$ de los entrevistados mostraron la variable de sensación térmica en la escala de "ni calor ni frío", el 36.8\% la escala de "regular" sensación de humedad, el 43.9\% la escala de "poca" en sensación de viento y el 59.6\% la escala de "sin radiación" en sensación de radiación. A la hora de hablar de la preferencia térmica la escala que resultó mayor fue "más frío" con el $36.8 \%$, la escala de "poca" un $42.1 \%$ para la preferencia de humedad y la escala de "media y poca" con el $28.1 \%$ respectivamente para la preferencia de viento y la escala de "sin cambio" en un $56.1 \%$ en la variable de preferencia de radiación. En lo que refiere a la aceptación de la temperatura la escala de "aceptable" fue la mayor con el $59.6 \%$ de los registros y la tolerancia de la temperatura fue la escala de "tolerable" en el $59.6 \%$ de los casos.

\begin{tabular}{|c|c|c|c|c|c|c|c|}
\hline & & $\begin{array}{l}\text { U05.01 } \\
\text { Temperatura } \\
\text { de bulbo seco }\end{array}$ & $\begin{array}{l}\text { U05.02 } \\
\text { Temperatura } \\
\text { de globo } \\
\text { negro }\end{array}$ & $\begin{array}{l}\text { U05.04 } \\
\text { Radiación } \\
\text { solar }\end{array}$ & $\begin{array}{l}\text { U05.05 } \\
\text { Humedad } \\
\text { relativa }\end{array}$ & $\begin{array}{l}\text { U05.07 } \\
\text { Velocidad } \\
\text { de viento }\end{array}$ & $\begin{array}{l}\text { U04.5 } \\
\text { Preferencia } \\
\text { térmica }\end{array}$ \\
\hline \multirow{3}{*}{$\begin{array}{l}\text { U05.01 Temperatura de bulbo } \\
\text { seco }\end{array}$} & Correlación de Pearson & 1 &, $766^{* *}$ & ,674 &,$- 863^{* *}$ & 0,097 & $-0,141$ \\
\hline & Sig. (bilateral) & & 0 & 0 & 0 & 0,473 & 0,296 \\
\hline & $\mathrm{N}$ & 57 & 57 & 57 & 57 & 57 & 57 \\
\hline \multirow{3}{*}{$\begin{array}{l}\text { U05.02 Temperatura de globo } \\
\text { negro }\end{array}$} & Correlación de Pearson &, $766^{* *}$ & 1 & $690^{* *}$ &,$- 747^{* *}$ & 0,129 & $-0,101$ \\
\hline & Sig. (bilateral) & 0 & & 0 & 0 & 0,338 & 0,453 \\
\hline & $\mathrm{N}$ & 57 & 57 & 57 & 57 & 57 & 57 \\
\hline \multirow{3}{*}{ U05.04 Radiación solar } & Correlación de Pearson &, $674^{* *}$ & $690^{* *}$ & 1 &,$- 629^{* *}$ & 0,081 &,$- 285^{*}$ \\
\hline & Sig. (bilateral) & 0 & 0 & & 0 & 0,547 & 0,032 \\
\hline & $\mathrm{N}$ & 57 & 57 & 57 & 57 & 57 & 57 \\
\hline \multirow{3}{*}{ U05.05 Humedad relativa } & Correlación de Pearson &,$- 863^{* *}$ &,$- 747^{* *}$ &,$- 629^{* *}$ & 1 & $-0,147$ & 0,171 \\
\hline & Sig. (bilateral) & 0 & 0 & 0 & & 0,276 & 0,204 \\
\hline & $\mathrm{N}$ & 57 & 57 & 57 & 57 & 57 & 57 \\
\hline \multirow{3}{*}{ U05.07 Velocidad de viento } & Correlación de Pearson & 0,097 & 0,129 & 0,081 & $-0,147$ & 1 & 0,086 \\
\hline & Sig. (bilateral) & 0,473 & 0,338 & 0,547 & 0,276 & & 0,526 \\
\hline & $\mathrm{N}$ & 57 & 57 & 57 & 57 & 57 & 57 \\
\hline \multirow{3}{*}{ U04.5 Preferencia térmica } & Correlación de Pearson & $-0,141$ & $-0,101$ &,$- 285^{*}$ & 0,171 & 0,086 & 1 \\
\hline & Sig. (bilateral) & 0,296 & 0,453 & 0,032 & 0,204 & 0,526 & \\
\hline & $\mathrm{N}$ & 57 & 57 & 57 & 57 & 57 & 57 \\
\hline
\end{tabular}

Tabla 2. Asociación de preferencia térmica con variables meteorológicas en exteriores periodo cálido.

** La correlación es significativa al nivel 0,01 (bilateral).

* La correlación es significante al nivel 0,05 (bilateral).

Fuente: Elaboración propia.
En lo que respecta al periodo frío en los espacios interiores el $47.1 \%$ de los entrevistados presentaron la variable de sensación térmica en la escala de "ni calor ni frío", el 38.2\% la escala de "nada" sensación de humedad y el 70.6\% la escala de "nada" en sensación de viento, con relación a la preferencia térmica, la escala que resultó mayor fue "más frío" con el $55.9 \%$, la escala de "nada y poca" con un $36.8 \%$ para la preferencia de humedad y la escala de "media" con un $44.1 \%$ para la preferencia de viento. En lo que refiere a la aceptación de la temperatura la escala de "aceptable" fue la mayor con el $57.4 \%$ de los registros y la tolerancia de la temperatura fue la escala de "tolerable" en el $52.9 \%$ de los casos.

Acerca de los registros del periodo frío en espacios exteriores, el $51.2 \%$ de los entrevistados mostraron la variable de sensación térmica en la escala de "ni calor ni frío", el $35.7 \%$ la escala de "poca" sensación de humedad, el $34.5 \%$ la escala de "poca" en sensación de viento y el 39.3\% la escala de "sin radiación" en sensación de radiación. En relación con la preferencia térmica la escala que resultó mayor fue "sin cambio" con el $45.2 \%$, la escala de "poca" un $39.3 \%$ para la preferencia de humedad y la escala de "media" con el $41.7 \%$ respectivamente para la preferencia de viento y la escala de "sin cambio" en un $51.2 \%$ en la variable de preferencia de radiación. En lo que refiere a la aceptación de la temperatura la escala de "aceptable" fue la mayor con el $60.7 \%$ de los registros y la tolerancia de la temperatura fue la escala de "tolerable" en el $70.2 \%$ de los casos. 


\begin{tabular}{|c|c|c|c|c|c|c|c|}
\hline & & $\begin{array}{l}05.01 \\
\text { Temperatura } \\
\text { de bulbo seco }\end{array}$ & $\begin{array}{l}05.02 \\
\text { Temperatura de } \\
\text { globo negro }\end{array}$ & \begin{tabular}{|l|} 
U05.04 \\
Radiación \\
solar
\end{tabular} & $\begin{array}{l}\text { U05.05 } \\
\text { Humedad } \\
\text { relativa }\end{array}$ & $\begin{array}{l}\text { U05.07 } \\
\text { Velocidad de } \\
\text { viento }\end{array}$ & $\begin{array}{l}\text { U04.9 } \\
\text { Aceptación } \\
\text { de la } \\
\text { temperatura }\end{array}$ \\
\hline \multirow{3}{*}{ U05.01 Temperatura de bulbo seco } & Correlación de Pearson & 1 &, $592^{* \star}$ & 0.193 & -0.038 &.$a$ & -0.051 \\
\hline & Sig. (bilateral) & & 0 & 0.114 & 0.758 & . & 0.68 \\
\hline & $\mathrm{N}$ & 68 & 67 & 68 & 68 & 68 & 68 \\
\hline \multirow{3}{*}{ U05.02 Temperatura de globo negro } & Correlación de Pearson &, $592^{* *}$ & 1 &, $439^{* \star}$ & -0.01 & .a & -0.07 \\
\hline & Sig. (bilateral) & 0 & & 0 & 0.935 & . & 0.575 \\
\hline & $\mathrm{N}$ & 67 & 67 & 67 & 67 & 67 & 67 \\
\hline \multirow{3}{*}{ U05.04 Radiación solar } & Correlación de Pearson & 0.193 &, $439^{* *}$ & 1 & 0.06 &.$a$ & -0.085 \\
\hline & Sig. (bilateral) & 0.114 & 0 & & 0.625 & . & 0.49 \\
\hline & $\mathrm{N}$ & 68 & 67 & 68 & 68 & 68 & 68 \\
\hline \multirow{3}{*}{ U05.05 Humedad relativa } & Correlación de Pearson & -0.038 & -0.01 & 0.06 & 1 &.$a$ & 0.167 \\
\hline & Sig. (bilateral) & 0.758 & 0.935 & 0.625 & & . & 0.173 \\
\hline & $\mathrm{N}$ & 68 & 67 & 68 & 68 & 68 & 68 \\
\hline \multirow{3}{*}{ U05.07 Velocidad de viento } & Correlación de Pearson &.$a$ &.$a$ &.$a$ & .a &.$a$ &.$a$ \\
\hline & Sig. (bilateral) & & & . & . & & \\
\hline & $\mathrm{N}$ & 68 & 67 & 68 & 68 & 68 & 68 \\
\hline \multirow{3}{*}{ U04.9 Aceptación de la temperatura } & Correlación de Pearson & -0.051 & -0.07 & -0.085 & 0.167 &.$a$ & 1 \\
\hline & Sig. (bilateral) & 0.68 & 0.575 & 0.49 & 0.173 & . & \\
\hline & $\mathrm{N}$ & 68 & 67 & 68 & 68 & 68 & 68 \\
\hline
\end{tabular}

Tabla 3. Asociación de aceptación de la temperatura con variables meteorológicas en interio-res periodo frío.

** La correlación es significativa al nivel 0,01 (bilateral).

a. No se puede calcular porque al menos una variable es constante.

Fuente: Elaboración propia.

\begin{tabular}{|c|c|c|c|c|c|c|c|}
\hline & & \begin{tabular}{|l|} 
U05.01 \\
Temperatura \\
de bulbo \\
seco
\end{tabular} & \begin{tabular}{|l|} 
U05.02 \\
Temperatura \\
de globo \\
negro
\end{tabular} & $\begin{array}{l}\text { U05.04 } \\
\text { Radiación } \\
\text { solar }\end{array}$ & $\begin{array}{l}\text { U05.05 } \\
\text { Humedad } \\
\text { relativa }\end{array}$ & $\begin{array}{l}\text { U05.07 } \\
\text { Velocidad } \\
\text { de viento }\end{array}$ & $\begin{array}{l}\text { U04.10 } \\
\text { Tolerancia } \\
\text { de la } \\
\text { temperatura }\end{array}$ \\
\hline \multirow{3}{*}{ U05.01 Temperatura de bulbo seco } & Correlación de Pearson & 1 &, $747^{* \star}$ & ,294* &,$- 923^{* *}$ & -0.117 & -0.029 \\
\hline & Sig. (bilateral) & & 0 & 0.013 & 0 & 0.292 & 0.792 \\
\hline & $\mathrm{N}$ & 83 & 83 & 71 & 83 & 83 & 83 \\
\hline \multirow{3}{*}{ U05.02 Temperatura de globo negro } & Correlación de Pearson &, $747^{\star \star}$ & 1 &, $656^{\star \star}$ &,$- 664^{* *}$ & 0.01 &,$- 259^{*}$ \\
\hline & Sig. (bilateral) & 0 & & 0 & 0 & 0.927 & 0.018 \\
\hline & $\mathrm{N}$ & 83 & 83 & 71 & 83 & 83 & 83 \\
\hline \multirow{3}{*}{ U05.04 Radiación solar } & Correlación de Pearson & ,294* &, $656^{* *}$ & 1 & -0.193 & 0.113 &,$- 297^{*}$ \\
\hline & Sig. (bilateral) & 0.013 & 0 & & 0.107 & 0.348 & 0.012 \\
\hline & $\mathrm{N}$ & 71 & 71 & 71 & 71 & 71 & 71 \\
\hline \multirow{3}{*}{ U05.05 Humedad relativa } & Correlación de Pearson &,$- 923^{* *}$ &,$- 664^{* *}$ & -0.193 & 1 & 0.126 & -0.026 \\
\hline & Sig. (bilateral) & 0 & 0 & 0.107 & & 0.255 & 0.814 \\
\hline & $\mathrm{N}$ & 83 & 83 & 71 & 83 & 83 & 83 \\
\hline \multirow{3}{*}{ U05.07 Velocidad de viento } & Correlación de Pearson & -0.117 & 0.01 & 0.113 & 0.126 & 1 & -0.046 \\
\hline & Sig. (bilateral) & 0.292 & 0.927 & 0.348 & 0.255 & & 0.683 \\
\hline & $\mathrm{N}$ & 83 & 83 & 71 & 83 & 83 & 83 \\
\hline \multirow{3}{*}{ U04.10 Tolerancia de la temperatura } & Correlación de Pearson & -0.029 &,$- 259^{*}$ &,$- 297^{*}$ & -0.026 & -0.046 & 1 \\
\hline & Sig. (bilateral) & 0.792 & 0.018 & 0.012 & 0.814 & 0.683 & \\
\hline & $\mathrm{N}$ & 83 & 83 & 71 & 83 & 83 & 83 \\
\hline
\end{tabular}

Tabla 4. Asociación de tolerancia de la temperatura con variables meteorológicas en exteriores durante el periodo frío.

** La correlación es significativa al nivel 0,01 (bilateral).

* La correlación es significante al nivel 0,05 (bilateral).

Fuente: Elaboración propia. 


\section{Discusión y conclusiones}

Con base en los resultados del estudio, se determina que el confort térmico en los espacios educativos es de importancia para que los usuarios puedan desempeñar sus funciones de una manera correcta. Se entiende que las condiciones de ambiente térmico en los interiores de una institución pueden ser controladas, a diferencia de los espacios exteriores que limitan la sensación de bienestar de un individuo. En lo que respecta a la correlación de Pearson en el periodo cálido en espacios interiores el rubro de sensación térmica representó una asociación significativa de nivel moderada con la variable de temperatura de bulbo seco y nivel débil con la temperatura de globo negro. La preferencia térmica refirió asociación significativa en nivel débil con la temperatura de bulbo seco y la temperatura de globo negro. La aceptación de la temperatura sucedió solo con la temperatura de bulbo seco en nivel de débil. La tolerancia de la temperatura solo fue significativa con la humedad relativa en el nivel de débil.

Asimismo, la sensación térmica tuvo una ligera asociación con la actividad metabólica y tipo de vestimenta y sobre la preferencia térmica, aceptación de la temperatura y tolerancia de la temperatura no tuvieron asociación significativa con las variables de usuario. Para el periodo cálido en espacios exteriores La correlación de Pearson en el rubro de sensación térmica representó una asociación de nivel significativo con la variable de temperatura de bulbo seco y nivel moderado con la temperatura de globo negro, radiación solar y humedad relativa. La preferencia térmica refirió asociación significativa en nivel débil con la radiación solar. La aceptación de la temperatura tuvo asociación débil con la temperatura de bulbo seco, temperatura de globo negro, humedad relativa y velocidad de viento. La tolerancia de la temperatura fue significativa en nivel débil con la temperatura de bulbo seco, temperatura de globo negro y humedad relativa.

En este rubro la sensación térmica tuvo asociación con la actividad metabólica y tipo de vestimenta en el nivel de débil, la preferencia térmica con la actividad metabólica en nivel de débil, la aceptación de la temperatura la actividad metabólica en nivel de débil y la tolerancia de la temperatura con la actividad metabólica en nivel de débil. En relación con el periodo frío en espacios interiores, la correlación de Pearson en el rubro de sensación térmica representó una asociación significativa de nivel débil con la variable de temperatura de globo negro. La preferencia térmica refirió asociación significativa en nivel débil con la temperatura de globo negro. La aceptación de la temperatura no tuvo asociación significativa con variables meteorológicas y la tolerancia de la temperatura solo fue significativa con la temperatura de globo negro en el nivel de débil. A diferencia de los resultados de periodo cálido en interiores, la sensación térmica, preferencia térmica, aceptación de la temperatura y tolerancia de la temperatura no tuvieron asociación significativa con la actividad metabólica y tipo de vestimenta.

Para concluir con lo relevante de los registros del periodo frío en exteriores, la correlación de Pearson en el rubro de sensación térmica representó una asociación significativa de nivel moderada con la variable de temperatura de bulbo seco y temperatura de globo negro y nivel débil con la humedad relativa. La preferencia térmica refirió asociación significativa en nivel moderado con la temperatura de bulbo seco y temperatura de globo negro, asociación débil con la radiación solar y humedad relativa. La aceptación de la temperatura no tuvo asociación con ninguna de las variables meteorológicas. La tolerancia de la temperatura fue significativa en nivel débil con la temperatura de globo negro y radiación solar. En este apartado la sensación térmica y la aceptación térmica no tuvieron asociación con la actividad metabólica y tipo de vestimenta, en cambio, la aceptación de la temperatura y la tolerancia de la temperatura tuvieron asociación significativa en nivel de débil con la actividad metabólica.

De acuerdo con los resultados obtenidos, se analiza que este estudio contribuye a la relación y adecuación entre el hombre y su entorno, a su vez, se habla también de la integración que resulta de la capacidad de satisfacer las necesidades humanas, sus expectativas de desarrollo y la convivencia en espacios educativos. Por otro lado, la sensación de confort térmico puede ser afectada por el nivel de actividad que desempeña una persona, así como su nivel de arropamiento en función del entorno ambiental, no obstante, esto resulta de interés no sólo cuando se trata de evaluar una situación, sino cuando se pretende transformar un ambiente térmico. 
Las conclusiones que se derivan del trabajo de investigación vinculan entre sí los temas tratados en el estudio. Estos son sensación térmica, preferencia térmica, aceptación de la temperatura y tolerancia de la temperatura en espacios interiores y exteriores de una institución educativa en una región con clima cálido semiseco. Se recomienda que el estudio presentado se considere para investigaciones futuras, en las cuales las variables y parámetros utilizados permitan crear estudios similares en la región de Sierra de Amula y la Costa Sur de Jalisco, México. Lo anterior para que pueda ser aprovechado por organismos municipales y permita mejorar las condiciones de los espacios educativos en cuanto a su habitabilidad y confort térmico se refiere.

\section{Referencias bibliográficas}

Asociación Española de Normalización. (2005). Ergonomía del ambiente térmico. Determinación de la tasa metabólica. (UNE 8996)

Bojórquez, G. (2010). Confort Térmico en Exteriores: Actividades en Espacios Recreativos en Clima Cálido Seco Extremo. (Tesis de Doctorado). Universidad de Colima, Colima, México. Obtenido de http://digesest.ucol.mx/tesis_posgrado/Pdf/ BOJORQUEZ_MORALES_GONZALO.pdf

Carranza,A., González, M., y Ojeda, R. (2016). Modelo de formación dual del Tecnológico Nacional de México. Revista de Investigación en Educación, 14(2), 170-183.

Coccolo, S., Kämpf, J., Scartezzini, J., y Pearlmutter, D. (2016). Outdoor human comfort and thermal stress: A comprehensive review on models and standards. Urban Climate 100 (18), 33-57.

Denisse, S. (2010). Norma Oficial Mexicana NOM-059-SEMARNAT-2010: Protección ambiental-especies nativas de México de flora y fauna silvestres-categorías de riesgo y especificaciones para su inclusión, exclusión o cambio-lista de especies en riesgo.

Godoy, A. (2012). El confort térmico adaptativo. Aplicación en la edificación en España. (Tesis de Maestría). Universidad Politécnica de Cataluña, Cataluña, España.

Gómez Azpeitia, G. (2007). El confort térmico: dos enfoques teóricos enfrentados. PALAPA, Revista de investigación científica en Arquitectura, 2(1) 44-57. UNEEN, I. 8996: (2005)..

Ochoa de la Torre, J., Marincic, I., y Alpuche, M. (2009). Análisis del confort climático para la planeación de sitios turísticos. In 5th International Conference Virtual City and Territory, Barcelona, 2, 3 and 4 June 2009 (pp. 481-488). Centre de Política de Sòl i Valoracions.

Organización Internacional de Normalización. (1989). Estrés térmico en personas con trabajo en ambientes cálidos. (ISO 7243).

Organización Internacional de Normalización. (1998). Ergonomics of the thermal environment-instruments for measuring physical quantities.(ISO 7726).

Organización Internacional de Normalización. (1995). Ergonomics of the thermal environment-Assessment of the influence of the thermal environment using subjective judgement scales. (ISO 10551).

Organización Internacional de Normalización. (2002). Ergonomics of the thermal environment-Instruments for measuring physical quantities. (ISO 7726).

Organización Internacional de Normalización. (2004). Ergonomics-Evaluation of Thermal Strain by Physiological Measurements (ISO 9886).

Organización Internacional de Normalización. (2005). Ergonomía del ambiente térmico. Determinación analítica e interpretación del bienestar térmico mediante el cálculo de los índices PMV y PPD y los criterios de bienestar térmico local. (ISO 7730).

Organización Internacional de Normalización. (2005). Ergonomía del ambiente térmico. Determinación analítica e interpretación del estrés térmico mediante el cálculo de la sobrecarga térmica estimada. (ISO 7933). 
Organización Internacional de Normalización. (2009). Ergonomics of the thermal environment-Estimation of thermal insulation and water vapor resistance of a clothing ensemble. (ISO 9920).

Organización Meteorológica Mundial. (2014). Guía de instrumentos y métodos de observación meteorológicos. Tiempo-Clima-Agua. OMM, Ginebra, Suiza.

Queiroz, S. (2016). Daytime thermal comfort in urban spaces: A field study in Brazil. Building and Environment, 107, 245-253.

Rein, M. (2013). El confort urbano en la ciudad y su evolución en los últimos años. Territorio y configuración de la ciudad. Agenda, 21.

Rincón, J., Fuentes, V., Bojórquez, G., y Ambriz, J. (2015). Efecto del clima local en la sensación térmica bajo condiciones controladas de laboratorio. Palapa, 3(18), 71-83.

Ruiz, M., y Correa, E. (2009). Confort térmico en espacios abiertos. Avances en Energías Renovables y Medio Ambiente, 13.

Rupp, R., Vásquez, N., y Lamberts, R. (2015). A review of human thermal comfort in the built environment. Energy and Buildings, 105, 178-205.

Salata, F., Golasi, I., Petitti, D., de Lieto Vollaro, E., Coppi, M., y de Lieto Vollaro, A. (2017). Relating microclimate, human thermal comfort and health during heat waves: An analysis of heat island mitigation strategies through a case study in an urban outdoor environment. Sustainable Cities and Society, 30, 79-96.

Vargas, M. (08 de mayo de 2017). Reserva de la Biosfera reconoce trabajo de brigadistas forestales. Obtenido de Letra Fría: http://letrafria.com/17990-2/

Yang, B., Olofsson, T., Nair, G., y Kabanshi, A. (2017). Outdoor thermal comfort under subarctic climate of north Sweden-A pilot study in Umeå. Sustainable cities and society, 28, 387-397. 